\title{
Indirect Effect of Insulin to Suppress Endogenous Glucose Production Is Dominant, Even with Hyperglucagonemia
}

\author{
Steven D. Mittelman, ${ }^{\star}$ You-Yin Fu, ${ }^{\star}$ Kerstin Rebrin, ${ }^{\ddagger}$ Garry Steil, ${ }^{\S}$ and Richard N. Bergman* \\ *Department of Physiology \& Biophysics, University of Southern California School of Medicine, Los Angeles, California 90033; \\ ${ }^{\ddagger}$ Minimed, Inc., Sylmar, California 91342; and ${ }^{\S}$ Joslin Diabetes Center, Boston, Massachusetts 02215
}

\begin{abstract}
Suppression of endogenous glucose production (EGP) is one of insulin's primary metabolic effects and failure of this action is a major contributor to fasting hyperglycemia of type 2 diabetes mellitus. Classically, insulin was thought to suppress the liver directly, via hyperinsulinemia in the portal vein. Recently, however, we and others have demonstrated that at least part, and possibly most of insulin's action to suppress EGP is normally mediated via an extrahepatic (i.e., indirect) mechanism. We have suggested that this mechanism involves insulin suppression of adipocyte lipolysis, leading to lowered FFA and reduced EGP ("Single Gateway Hypothesis"). Previous studies of the indirect insulin effect from this laboratory were done under conditions of lowered portal glucagon. Because of the possibility that the direct (i.e., portal) effect of insulin may have been underestimated with hypoglucagonemia, these studies examined the relative importance of portal insulin, versus peripheral insulin (administered at one-half the dose to equalize peripheral insulin levels) at four rates of portal glucagon infusion: $0,0.65$ (under-), 1.5 (basal-), and $3.0 \mathrm{ng} / \mathrm{kg}$ per min (over-replacement). Portal versus peripheral insulin suppressed steady-state EGP to the same extent (52\%), confirming that the primary effect of insulin to suppress EGP is via the peripheral mechanism. This conclusion was maintained regardless of portal glucagonemia, although there was some evidence for an increase in the direct insulin effect at hyperglucagonemia. The indirect effect of insulin is the primary mechanism of steady-state EGP suppression under normal conditions. The direct effect increases with hyperglucagonemia; however, the indirect effect remains predominant even under those conditions. (J. Clin. Invest. 1997. 100:3121-3130.) Key words: liver • NIDDM • free fatty acids • metabolism • turnover
\end{abstract}

\section{Introduction}

As insulin is secreted directly into the portal vein, it is reasonable to assume that it acts directly to suppress endogenous glu-

Address correspondence to Richard N. Bergman, University of Southern California School of Medicine, Department of Physiology and Biophysics, MMR626, 1333 San Pablo St., Los Angeles, CA 90033. Phone: 213-342-1920; FAX: 213-342-1918; E-mail: rbergman@ syntax.hsc.vsc.edu

Received for publication 29 April 1997 and accepted in revised form 16 October 1997.

J. Clin. Invest.

(C) The American Society for Clinical Investigation, Inc. 0021-9738/97/12/3121/10 \$2.00

Volume 100, Number 12, December 1997, 3121-3130

http://www.jci.org cose production (EGP). ${ }^{1}$ However, this view was incongruent with the failure of insulin to consistently suppress EGP in vitro (1-3). Several recent studies from our laboratory and others have shown that much of insulin's action to suppress the liver is in fact an indirect, systemic effect (4-10). This effect could be mediated by insulin-sensitive metabolites such as free fatty acids, glucagon, and/or gluconeogenic precursors (lactate, glycerol, amino acids). There is also evidence that glucose production by the kidneys is regulated by systemic insulin levels (11). Ader et al. demonstrated that the majority of EGP is regulated by systemic insulin levels (4), and her results have been corroborated by Giacca et al. (9) and Rebrin et al. (5).

In contrast to these findings, a direct effect of insulin on glucose output has been clearly demonstrated in the presence of high glucagon levels in both perfused liver $(2,12)$ and isolated hepatocytes $(1,13)$. In addition, several in vivo studies have concluded that portal insulin is a major controller of EGP $(10,14-18)$. The discrepancy in the literature as to the magnitude of the direct effect of insulin could be related to differences in glucagon levels among these studies. An "antiglucagon" effect of insulin might not be obvious in studies in which glucagon is suppressed (5) or not matched between protocols (9). Furthermore, many studies comparing portal and one-half dose systemic routes of insulin delivery may not have accurately matched peripheral insulin levels due to the variability of hepatic extraction (19) and/or incomplete suppression of the pancreas by somatostatin or insulin. This potential for imprecision makes it difficult to control possible secondary modulators of insulin action, such as FFA or glucagon, which could be exquisitely sensitive to peripheral insulin levels and confound comparisons of portal and peripheral routes of insulin administration. Finally, it is difficult to isolate the direct and indirect effects of insulin since both may be present to some degree regardless of the route of insulin infusion.

A recent study by Lewis et al. (20) concluded that the direct effect of insulin to suppress EGP is enhanced by glucagon. Lewis and coworkers' study compared tolbutamide-induced insulin secretion with insulin infused systemically at the same rate. Since the liver extracts on a single pass $\sim 50 \%$ of the insulin in the portal vein, neither systemic nor portal insulin levels were matched in Lewis' study. Because of differing peripheral insulin, it is likely that FFA, glucagon, and other metabolites sensitive to systemic insulin levels would not have been matched, making comparisons between portal and peripheral protocols difficult to interpret (compare with reference 4). Giacca et al. performed two studies comparing portal vs. one-half systemic insulin infusion, one with and one without a high glucagon infusion $(9,21)$. The study done with high glucagon detected a direct effect of insulin while the other study

1. Abbreviations used in this paper: EGP, endogenous glucose production; NIDDM, non-insulin-dependent diabetes mellitus. 
did not. This study provided direct evidence that the direct effect of insulin to suppress EGP is dependent on glucagon.

Since the direct and indirect effects of insulin are currently under intensive debate, we felt that a comprehensive study was necessary to examine the direct effect of insulin on glucose production at different physiologic glucagon levels. In the present study, endogenous secretion of glucagon was suppressed with somatostatin, and glucagon was replaced at four different concentrations: zero, underreplacement, replacement, and overreplacement. Insulin was infused portally, or peripherally at half of the portal rate, to match systemic levels while attaining very different portal vein levels $(4,5)$. An insulin dose was chosen that would yield approximately two-thirds suppression of EGP under the conditions of our experiments. The direct and indirect effects of insulin were examined by comparing portal and systemic insulin infusions (at one-half the portal rate) at each glucagon dose.

\section{Methods}

Animals. Experiments were performed on seven male mongrel dogs $(26.6 \pm 1.1 \mathrm{~kg})$. The maintenance and surgery on the animals have been previously described (5). Chronic catheters were implanted at least $7 \mathrm{~d}$ before experiments. One catheter was inserted into the jugular vein and advanced to the right atrium for sampling of central venous blood. A second catheter was placed in the portal vein $4 \mathrm{~cm}$ upstream from the porta hepatis for portal infusions of glucagon and insulin. We have previously shown that slow infusions given at this site are equally distributed among the lobes of the liver (22). A third catheter was placed in the femoral vein and advanced to the inferior vena cava for the infusion of tracer, somatostatin, and insulin.

Experimental protocol. Each dog underwent eight different euglycemic clamp protocols; each protocol was separated by at least $5 \mathrm{~d}$. Glucagon was infused portally in each experiment at one rate: either $0.00,0.65,1.50$, or $3.00 \mathrm{ng} / \mathrm{kg}$ per min (porcine glucagon; Sigma Chemical Co., St. Louis, MO). The $1.50 \mathrm{ng} / \mathrm{kg}$ per min glucagon dose was determined to reestablish basal measured glucagon levels in pilot experiments. Insulin was infused either portally at a rate of $0.8 \mathrm{mU} / \mathrm{kg}$ per min or peripherally at half the portal rate $(0.4 \mathrm{mU} / \mathrm{kg}$ per min, regular insulin; Novo-Nordisk, Copenhagen, Denmark). Five animals completed all eight experiments, one received the zero, low, and high glucagon doses, and one received only the high glucagon dose. Experiments consisted of a 150-min equilibration period during which a primed infusion of HPLC-purified $3-{ }^{3} \mathrm{H}$ D-glucose was given $(25 \mu \mathrm{Ci}+$ $0.25 \mu \mathrm{Ci} / \mathrm{min}$; NEN Research Products Du Pont, Boston, MA), followed by the clamp period (0-270 min). Basal samples were taken every 10 min from $t=-60$ to 0 . At time $t=0$, infusions of somatostatin $(1.0 \mu \mathrm{g} / \mathrm{kg}$ per min; Bachem California, Torrance, CA $)$, insulin, and glucagon were started. All solutions were infused at a flow rate of $0.25 \mathrm{ml} / \mathrm{min}$. Glucose was clamped at basal by a variable glucose infusion labeled with $3-{ }^{3} \mathrm{H}$ D-glucose $(1.8 \mu \mathrm{Ci} / \mathrm{gram})$ to maintain specific activity approximately constant (23). Blood samples for on-line glucose assay were taken every $5 \mathrm{~min}$ for the first hour of the clamp, and then every $10 \mathrm{~min}$ for the remainder of the experiment. Glucose was measured on all samples, while insulin, glucagon, tracer, and FFA were assayed on all samples between $t=-60$ and $100 \mathrm{~min}$, and every 20 min until the end of the experiment. Samples for glucose, insulin, and tracer assay were collected in tubes coated with lithium fluoride and heparin. Samples for glucagon assay were collected in Trasylol (aprotinin; $75 \mu \mathrm{l} / \mathrm{ml}$ blood; Miles Inc., Kankakee, IL), while those for assay of FFA were collected in EDTA and paraoxon (Sigma Chemical Co.) to suppress lipoprotein lipase (24). Samples for FFA, glucose, and tracer were immediately centrifuged and the plasma separated and kept on ice for processing that day. Plasma for insulin assay was stored at $-20^{\circ} \mathrm{C}$, and plasma for glucagon assay was stored at $-70^{\circ} \mathrm{C}$.
Assays. Glucose was measured immediately after sampling with a YSI 2700 autoanalyzer (Yellow Springs Instrument Co., Yellow Springs, OH). Samples for tracer assay were deproteinized using barium hydroxide and zinc sulfate. The supernatants were then evaporated in a vacuum, reconstituted in water, and counted in Ready Safe scintillation fluid (Beckman liquid scintillation counter; Beckman Instruments, Fullerton, CA). Tracer infusates were processed identically to plasma samples. Insulin was measured by an ELISA originally developed for human serum or plasma by Novo-Nordisk and adapted for dog plasma. The method is based on two murine monoclonal antibodies that bind to different epitopes on insulin, but not to proinsulin. Materials for the insulin assay, including the dog standard, were kindly provided by Novo-Nordisk. Glucagon was measured using a Linco RIA kit (Kit \#GL-32K; Linco Research, Inc., St. Charles, MO) with glucagon standards from Novo-Nordisk. Free fatty acids were measured using a kit from Wako (NEFA C; Wako Pure Chemical Industries, Richmond, VA), which utilizes a colorometric assay based on the acylation of coenzyme-A.

Calculations. Basal values are defined as the average of seven samples ( -60 to $0 \mathrm{~min}$ ) and steady-state as the average of 10 samples (120-270 min). EGP was calculated using Steele's model with a labeled glucose infusion as detailed previously (23). Katz et al. have demonstrated the equivalence of infusing labeled glucose into an artery and sampling for specific activity in the vena cava vs. infusing into a vein and sampling from an artery (25). Since glucose specific activity should not change across tissue beds that do not produce glucose, arterial and venous tracer infusions should yield similar mixed venous blood specific activities. Thus, the venous/mixed venous mode of tracer infusion/sampling used in the present study should give identical results to the arterial/mixed venous and the venous/arterial modes.

Portal insulin levels were estimated using the equation (4):

$\mathrm{I}_{\mathrm{PO}}=\mathrm{I}_{\mathrm{PE}}+\frac{\mathrm{INF}_{\mathrm{PO}}}{\mathrm{PPF}}$

where $\mathrm{I}_{\mathrm{PO}}$ and $\mathrm{I}_{\mathrm{PE}}$ represent portal and systemic (i.e., mixed venous) insulin levels, respectively, $\mathrm{INF}_{\mathrm{PO}}$ is the portal insulin infusion rate (zero during peripheral infusion protocols), and PPF is portal plasma flow, assumed to be $20 \mathrm{ml} / \mathrm{min} / \mathrm{kg}$ ( $500 \mathrm{ml} / \mathrm{min}$ normalized for weight, reference 26). Basal portal insulin levels were estimated for each experiment from the ratio:

$\frac{\text { Ins }_{\text {Basal, PO }}}{\mathrm{Ins}_{\text {Basal, PE }}}=\frac{\text { Ins }_{\text {SS, PO }}}{\mathrm{Ins}_{\mathrm{SS}, \mathrm{PE}}}$

where the steady-state insulin levels represent those from the portal infusion experiment performed on that animal at that glucagon dose. This calculation assumes similar steady-state insulin clearance rates during basal and insulin infusion periods in the same dog on both experimental days. Hepatic insulin levels were calculated assuming a $28 \%$ arterial vascularization of the liver (26). The direct and indirect effects of insulin on EGP were calculated using multiple linear regression with the equation:

$\Delta$ EGP $=\alpha_{1} \times \Delta$ systemic insulin $+\alpha_{2} \times \Delta$ hepatic insulin .

This method assumes that the change in EGP is a linear function of the changes in hepatic and/or systemic insulin levels. The $\alpha_{1}$ and $\alpha_{2}$ parameters represent the relative effect of systemic and hepatic insulin, respectively, to suppress EGP. The percent direct effect of insulin to suppress EGP is then defined as:

$\frac{\alpha_{2}}{\alpha_{1}+\alpha_{2}} \times 100 \%$.

The time to half-maximal suppression of EGP $\left(\mathrm{T}_{1 / 2}\right)$ and the integrated endogenous glucose production during the first $120 \mathrm{~min}$ of the clamp $\left(\mathrm{AUC}_{120}\right)$ were calculated to examine the dynamics of EGP 

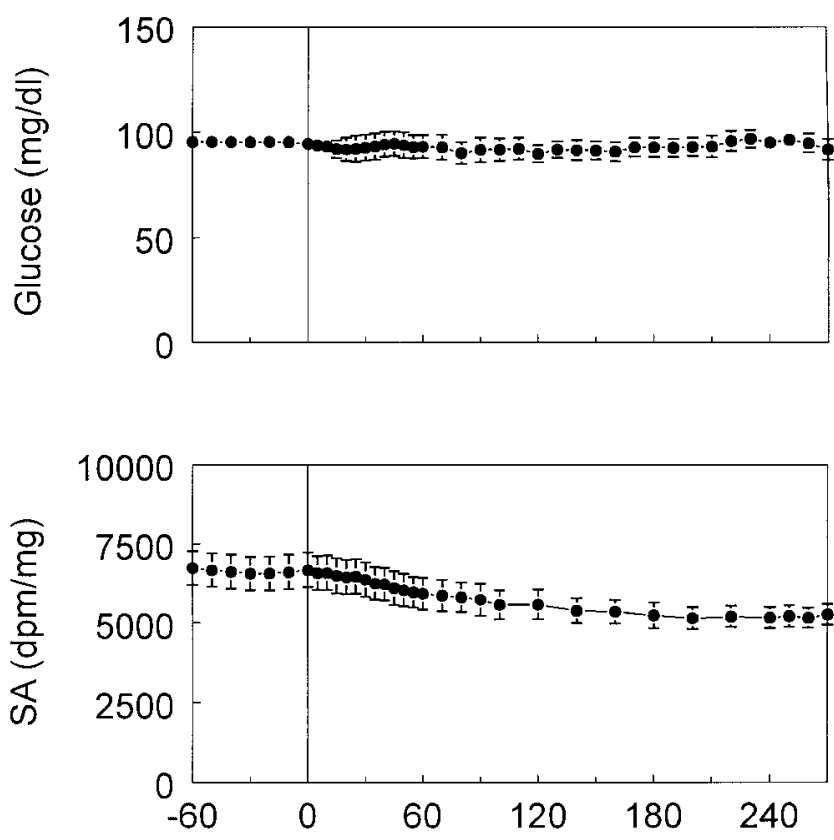

Figure 1. Average glucose (top) and glucose specific activity (bottom) during euglycemic clamps, pooled across all experimental protocols.

suppression during the clamps. The $\mathrm{T}_{1 / 2}$ parameter was calculated on MLAB (Civilized Software, Bethesda, MD) implemented on an IBM compatible computer using smoothed EGP data. Multiple linear regression was also performed on MLAB.

Statistics. Values are reported as mean \pm SEM. A two-factor repeated ANOVA (insulin infusion route vs. glucagon dose) was used to compare the effects of both insulin infusion route and glucagon dose on basal and steady-state values of insulin, glucagon, FFA, and
EGP, as well as the change in EGP, the $\mathrm{T}_{1 / 2}$ of EGP suppression, and the $\mathrm{AUC}_{120}$. A stratified analysis was then performed with a one-factor repeated ANOVA, comparing portal vs. peripheral infusion effects on EGP at each glucagon dose, which allowed adjustment for differences in systemic insulin levels between experiments. All statistical calculations were performed on SAS (Cary, NC) implemented on an IBM compatible computer. The quantification of the direct vs. indirect effects of insulin are presented as point estimates, and therefore statistical analysis of these values were not performed. These values are reported as estimate \pm standard error of estimate.

\section{Results}

Glucose and specific activity. Basal glucose averaged 95.4土 $0.7 \mathrm{mg} / \mathrm{dl}$ and was clamped at this level during insulin infusion (average coefficient of variation [CV] of $9 \%$, Fig. 1). Glucose specific activity fell slowly and moderately by $19 \pm 6 \%$ throughout the clamps, due to underlabeled variable glucose infusion (dogs on average exhibited less hepatic insulin sensitivity than expected). However, the specific activity remained constant throughout the steady-state period with a mean $\mathrm{CV}$ of $7 \%$. Hother-Nielsen et al. and Fisher et al. have shown that such slow changes in specific activity still yield accurate EGP results $(27,28)$.

Insulin. Basal systemic insulin in all dogs was $62 \pm 5 \mathrm{pM}$, and this value was not different among protocols $(P>0.05$, ANOVA, Table I and Fig. 2). Calculated basal hepatic insulin levels averaged $141 \pm 9 \mathrm{pM}$ in all groups except for the replacement glucagon group, where these values were significantly lower $(P<0.01$, ANOVA) partly due to the high fasting insulin level of one animal which did not undergo the replacement glucagon protocols $(P>0.05$ when this dog is not included in the analysis). As predicted from the experimental design, hepatic insulin levels were dramatically higher during portal infusion than one-half dose peripheral infusion ( $307 \pm 5$ vs. $162 \pm 4$

Table I. Glucose, Glucose Specific Activity, and Hormone Levels

\begin{tabular}{|c|c|c|c|c|c|c|c|c|}
\hline \multirow[b]{2}{*}{ Insulin } & \multicolumn{2}{|c|}{ No glucagon } & \multicolumn{2}{|c|}{ Low glucagon } & \multicolumn{2}{|c|}{ Replacement glucagon } & \multicolumn{2}{|c|}{ High glucagon } \\
\hline & $\mathrm{PO}$ & PE & $\mathrm{PO}$ & PE & $\mathrm{PO}$ & PE & $\mathrm{PO}$ & PE \\
\hline \multicolumn{9}{|l|}{ Glucose (mg/dl) } \\
\hline Basal & $95 \pm 3$ & $96 \pm 2$ & $96 \pm 2$ & $95 \pm 1$ & $94 \pm 2$ & $95 \pm 1$ & $95 \pm 2$ & $96 \pm 2$ \\
\hline SS & $94 \pm 3$ & $95 \pm 2$ & $91 \pm 2$ & $92 \pm 2$ & $94 \pm 2$ & $97 \pm 3$ & $91 \pm 3$ & $94 \pm 1$ \\
\hline \multicolumn{9}{|c|}{ Glucose SA (dpm/mg) } \\
\hline Basal & $6626 \pm 452$ & $6597 \pm 424$ & $6529 \pm 330$ & $6304 \pm 576$ & $6948 \pm 421$ & $6692 \pm 497$ & $6228 \pm 630$ & $6515 \pm 563$ \\
\hline SS & $5947 \pm 457$ & $5230 \pm 311$ & $5847 \pm 236$ & $5114 \pm 264$ & $5296 \pm 227$ & $4846 \pm 250$ & $5168 \pm 277$ & $4843 \pm 258$ \\
\hline \multicolumn{9}{|c|}{ Systemic Insulin (pM) } \\
\hline Basal & $82 \pm 23$ & $69 \pm 14$ & $61 \pm 13$ & $63 \pm 11$ & $48 \pm 15$ & $43 \pm 8$ & $62 \pm 12$ & $63 \pm 10$ \\
\hline SS & $127 \pm 14$ & $161 \pm 7$ & $123 \pm 9$ & $161 \pm 7$ & $139 \pm 11$ & $164 \pm 12$ & $150 \pm 7$ & $161 \pm 11$ \\
\hline \multicolumn{9}{|c|}{ Hepatic Insulin (pM) } \\
\hline Basal & $186 \pm 39$ & $161 \pm 27$ & $145 \pm 27$ & $151 \pm 20$ & $105 \pm 29$ & $97 \pm 17$ & $131 \pm 23$ & $135 \pm 19$ \\
\hline SS & $300 \pm 14$ & $161 \pm 7$ & $295 \pm 9$ & $161 \pm 7$ & $312 \pm 11$ & $164 \pm 12$ & $323 \pm 7$ & $161 \pm 11$ \\
\hline \multicolumn{9}{|c|}{ Glucagon (pg/ml) } \\
\hline Basal & $78 \pm 12$ & $99 \pm 11$ & $96 \pm 11$ & $92 \pm 11$ & $83 \pm 17$ & $77 \pm 9$ & $92 \pm 15$ & $106 \pm 19$ \\
\hline SS & $45 \pm 6$ & $45 \pm 6$ & $72 \pm 11$ & $70 \pm 7$ & $94 \pm 7$ & $93 \pm 8$ & $138 \pm 10$ & $165 \pm 17$ \\
\hline \multicolumn{9}{|l|}{ FFA $(\mu \mathrm{M})$} \\
\hline Basal & $522 \pm 64$ & $551 \pm 109$ & $551 \pm 53$ & $449 \pm 57$ & $563 \pm 108$ & $571 \pm 86$ & $454 \pm 91$ & $498 \pm 50$ \\
\hline SS & $117 \pm 34$ & $68 \pm 25$ & $81 \pm 23$ & $88 \pm 17$ & $77 \pm 29$ & $94 \pm 28$ & $89 \pm 19$ & $102 \pm 22$ \\
\hline
\end{tabular}

Basal and steady-state $(S S)$ hormone levels for euglycemic clamps with insulin infused portally $(0.8 \mathrm{mU} / \mathrm{min} \mathrm{per} \mathrm{kg}, P O)$ or peripherallly $(0.4 \mathrm{mU} / \mathrm{min}$ per kg, $P E$ ). Glucagon was suppressed with somatostatin and infused portally at one of four doses (zero, $0.65,1.5$, or $3.0 \mathrm{ng} / \mathrm{kg} \mathrm{per} \mathrm{min).}$ 


\section{Systemic Insulin Levels}

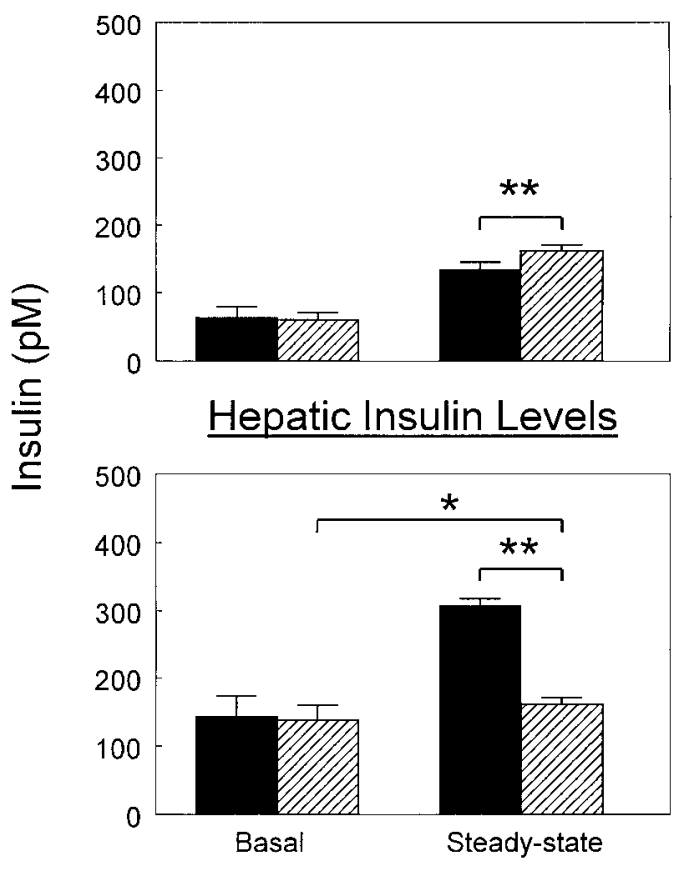

Figure 2. Basal and steady-state insulin levels achieved during portal $(0.8 \mathrm{mU} / \mathrm{kg}$ per min, solid bars $)$ and systemic $(0.4 \mathrm{mU} / \mathrm{kg}$ per min, hatched bars) insulin infusion. Systemic (top) and calculated hepatic (bottom) insulin levels are shown. ( $* * P<0.01$ portal vs. systemic infusion, ${ }^{*} P<0.05$ steady-state vs. basal)

pM). Despite the large differences in portal insulin concentrations, steady-state systemic insulin levels were almost the same between portal and peripheral infusions, differing by only $17 \%$ ( $135 \pm 5$ vs. $162 \pm 4 \mathrm{pM}, P=0.01$ ). This difference was due to an overall hepatic extraction $>50 \%(58 \pm 4 \%)$. Hepatic insulin levels rose slightly over basal during systemic insulin infusion $(162 \pm 4$ vs. $138 \pm 11 \mathrm{pM}, P<0.05)$. There was no significant effect of glucagon dose nor an interaction of glucagon dose and insulin infusion route to affect steady-state systemic or hepatic insulin levels ( $P>0.05$, ANOVA).

Glucagon. Basal systemic glucagon averaged $90 \pm 5 \mathrm{pg} / \mathrm{ml}$, and did not differ among protocols $(P>0.05$, Fig. 3, Table I). Systemic glucagon levels reached steady-state values of $71 \pm 6$, $94 \pm 5$, and $151 \pm 10 \mathrm{pg} / \mathrm{ml}$ during the $0.65,1.50$, and $3.00 \mathrm{ng} / \mathrm{kg}$ per min infusions, respectively. When glucagon was not replaced, plasma levels fell $\sim 50 \%$ to $45 \pm 4 \mathrm{pg} / \mathrm{ml}$. Glucagon level increased linearly with glucagon infusion rate $(r=0.86$, $P=0.0001)$. Infusion of $1.50 \mathrm{ng} / \mathrm{kg}$ per min yielded a measured steady-state level not different from basal ( $94 \pm 5$ vs. $90 \pm 5, P=$ $0.4)$.

FFA. Basal FFA were $516 \pm 27 \mu \mathrm{M}$, and this value did not differ among protocols $(P>0.05$, Table I). Insulin infusion caused FFA to fall within the first $30 \mathrm{~min}$ to an average value of $91 \pm 8 \mu \mathrm{M}$, and there was no difference between route of insulin infusion or glucagon dose $(P>0.05$, Fig. 4$)$.

Steady-state EGP. To compare the effects of hepatic and systemic insulin to suppress the liver, EGP was measured during portal and one-half dose peripheral insulin infusions, which should match systemic insulin levels while achieving very dif- ferent hepatic levels (Fig. 2). EGP values are reported in Table II and Fig. 5. Basal EGP averaged $2.87 \pm 0.08 \mathrm{mg} / \mathrm{kg}$ per min, and was not different between protocols $(P>0.05)$. On average EGP fell by $52 \pm 3 \%$ to a value of $1.38 \pm 0.09 \mathrm{mg} / \mathrm{kg}$ per min. Glucagon level did not affect steady-state EGP or the change from basal to steady-state $(\triangle \mathrm{EGP})$ with either route of insulin infusion $(P>0.05$ for both of these comparisons). There was a tendency for portal insulin infusion to yield greater suppression of EGP $(-1.66 \pm 0.09 \mathrm{mg} / \mathrm{kg}$ per min, or $57 \pm 3 \%$ suppression) than systemic insulin infusion $(-1.30 \pm 0.13 \mathrm{mg} / \mathrm{kg}$ per min, $47 \pm 5 \%$ suppression), despite lower systemic insulin concentrations during portal infusion; however, this effect did not reach statistical significance $(P=0.07)$. Likewise, steadystate EGP levels were not significantly different between routes of insulin infusion $(1.27 \pm 0.13$ vs. $1.50 \pm 0.13 \mathrm{mg} / \mathrm{kg}$ per min, portal vs. systemic infusion, $P=0.10$ ). When broken down by glucagon dose, the average decline in EGP from basal to steadystate is greater with portal than peripheral insulin infusion at each glucagon dose, although it was significantly greater only at the highest glucagon dose $(P=0.01$, stratified ANOVA corrected for systemic insulin level). Thus at the zero, low, and replacement glucagon doses, portal insulin infusion did not suppress steady-state EGP significantly beyond that induced by

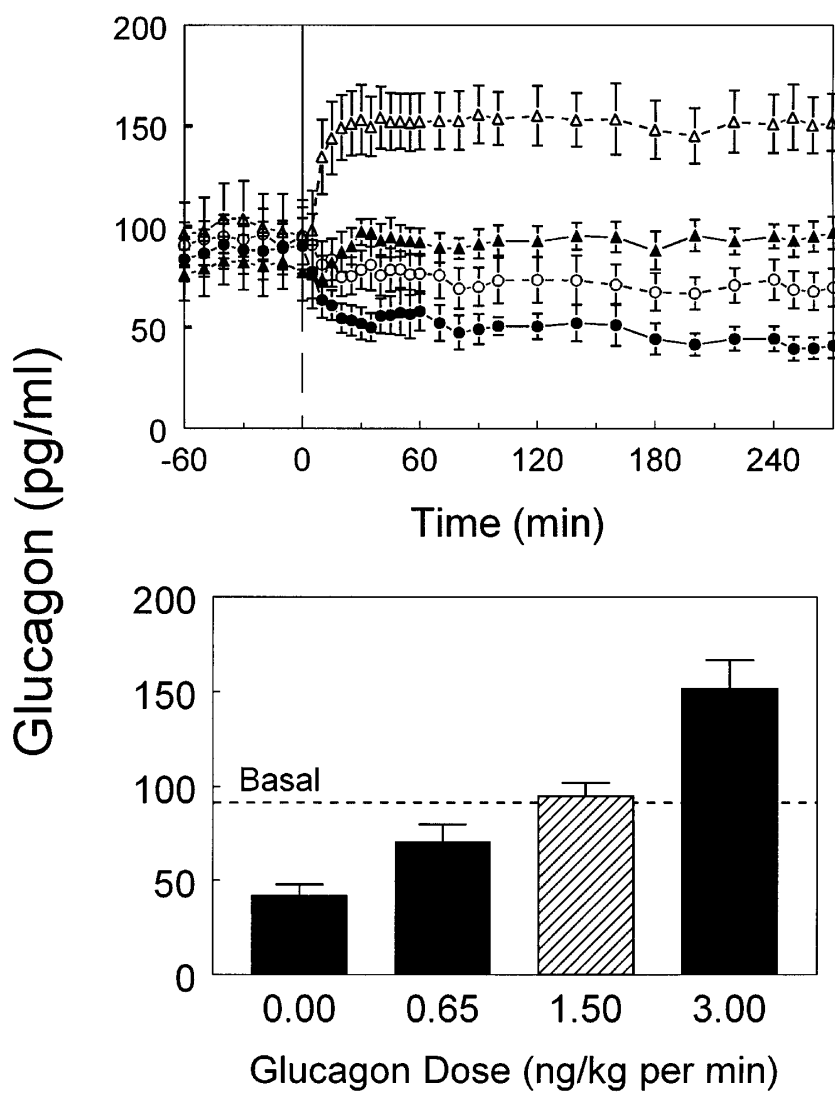

Figure 3. Dynamic glucagon profiles (top) and steady-state values (bottom). Dynamics are shown for the protocol where glucagon was suppressed and not replaced (closed circles), and with low (open circles), replacement (closed triangles), and high (open triangles) glucagon infusion rates. Steady-state glucagon level achieved during the replacement infusion (hatched bar) is not significantly different from the basal level pooled from all experiments. 

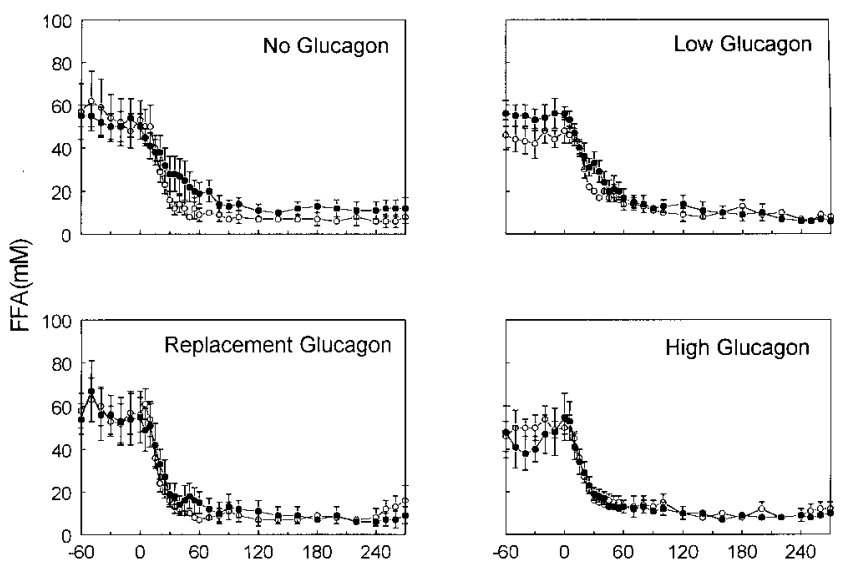

Time (min)

Figure 4. FFA profiles during each of the four glucagon doses. Portal (closed circles) and systemic (open circles) insulin infusion protocols are compared.

systemic infusion, even though hepatic insulin levels during portal infusion were nearly twice those attained during systemic infusion. Only at the highest glucagon dose was there an apparently greater suppression of steady-state EGP with portal than with systemic insulin infusion.

EGP dynamics. The $\mathrm{AUC}_{120}$ was calculated to examine insulin's effect to counter glucagon stimulation of EGP during the 0-120-min dynamic phase, since glucagon is known to have an evanescent effect (29-31). This value represents the total amount of glucose produced during the first $120 \mathrm{~min}$ of the clamp. The $\mathrm{AUC}_{120}$ showed a significant trend of increasing with glucagon dose with either route of insulin infusion, but especially with systemic insulin infusion $(P<0.005$ for systemic infusion, $P<0.05$ for portal, Table II), implying that portal insulin suppressed glucagon's dynamic effect to some degree. When portal and systemic routes of insulin were compared at each glucagon dose, the $\mathrm{AUC}_{120}$ was significantly lower (i.e., EGP was more suppressed during the dynamic period) with portal infusion than peripheral at the low, replacement, and high glucagon doses, but not at the zero glucagon dose $(P<$ 0.05 for portal vs. peripheral at 0.65 dose, $P=0.01$ for 1.5 and 3.0 glucagon doses). However, when the $\mathrm{AUC}_{120}$ was adjusted for systemic insulin in the stratified analysis, the significance
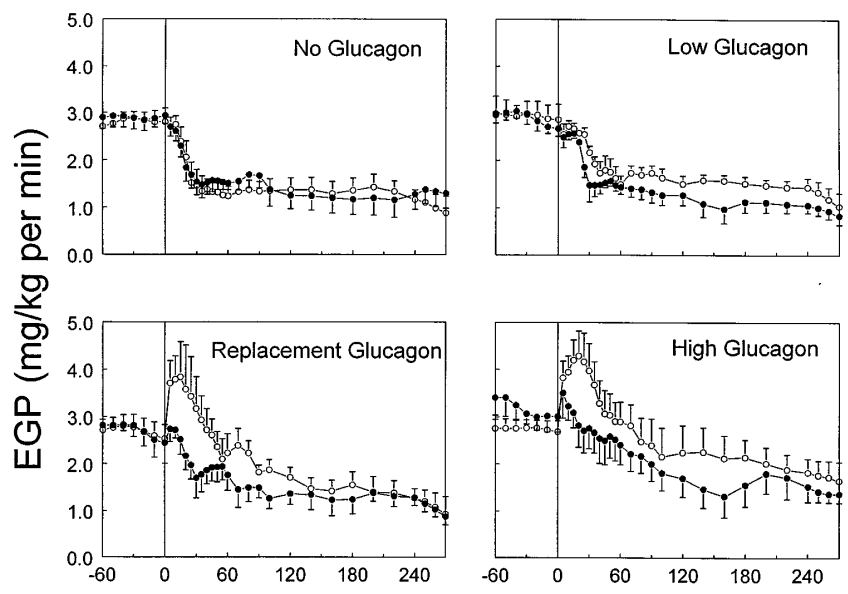

Time $(\min )$

Figure 5. Endogenous glucose production profiles during each of the four glucagon doses. Portal (closed circles) and systemic (open circles) insulin infusion protocols are compared.

was lost at all except the high glucagon dose $(P<0.05$ at high dose), due to the higher systemic insulin levels during peripheral insulin infusion at the lower three glucagon infusion rates. There was a tendency for the $T_{1 / 2}$ of EGP suppression to be lower with portal than systemic insulin infusion (i.e., faster suppression) with increasing glucagon dose, but this trend was not statistically significant $(P=0.12)$, probably due to the variability of this measurement.

Direct and indirect effects of insulin. The direct effect of portal insulin to suppress EGP was determined from multiple linear regression considering the change in EGP as a function of the changes in systemic vs. hepatic insulin (see Methods). When glucagon was not replaced, the direct effect represented only $11 \pm 19 \%$ of its total effect to lower EGP (Table III and Fig. 6). At the low and replacement glucagon doses, the direct effect of insulin accounted for $24 \pm 11$ and $21 \pm 17 \%$ of its total effect, respectively. At the highest glucagon dose, the direct effect of insulin represented $44 \pm 17 \%$ of its total effect. Similar results were also obtained when linear regressions were done considering the correlations from all eight protocols within each dog (not shown). When linear regression was performed using calculated portal insulin levels rather than hepatic (which would

Table II. Endogenous Glucose Production

\begin{tabular}{|c|c|c|c|c|c|c|c|c|}
\hline \multirow[b]{2}{*}{ Insulin } & \multicolumn{2}{|c|}{ No glucagon } & \multicolumn{2}{|c|}{ Low glucagon } & \multicolumn{2}{|c|}{ Replacement glucagon } & \multicolumn{2}{|c|}{ High glucagon } \\
\hline & $\mathrm{PO}$ & $\mathrm{PE}$ & $\mathrm{PO}$ & $\mathrm{PE}$ & $\mathrm{PO}$ & $\mathrm{PE}$ & $\mathrm{PO}$ & $\mathrm{PE}$ \\
\hline Basal EGP (mg/kg per min) & $2.90 \pm 0.22$ & $2.81 \pm 0.18$ & $2.88 \pm 0.13$ & $2.94 \pm 0.29$ & $2.70 \pm 0.22$ & $2.70 \pm 0.25$ & $3.17 \pm 0.31$ & $2.75 \pm 0.17$ \\
\hline SS EGP $(\mathrm{mg} / \mathrm{kg}$ per min $)$ & $1.25 \pm 0.29$ & $1.23 \pm 0.24$ & $1.05 \pm 0.20$ & $1.41 \pm 0.16$ & $1.22 \pm 0.20$ & $1.34 \pm 0.22$ & $1.51 \pm 0.32$ & $1.91 \pm 0.34$ \\
\hline$\Delta$ EGP (mg/kg per min) & $-1.65 \pm 0.15$ & $-1.58 \pm 0.19$ & $-1.83 \pm 0.16$ & $-1.53 \pm 0.28$ & $-1.48 \pm 0.11$ & $-1.36 \pm 0.25$ & $-1.65 \pm 0.24$ & $-0.84 \pm 0.24$ \\
\hline \% Suppression of EGP & $-57 \pm 5 \%$ & $-56 \pm 7 \%$ & $-64 \pm 6 \%$ & $-52 \pm 10 \%$ & $-55 \pm 4 \%$ & $-50 \pm 9 \%$ & $-52 \pm 8 \%$ & $-31 \pm 9 \%$ \\
\hline $\mathrm{T}_{1 / 2}(\mathrm{~min})$ & $16.9 \pm 2.3$ & $23.5 \pm 4.1$ & $27.2 \pm 3.7$ & $29.5 \pm 6.5$ & $58.3 \pm 20.3$ & $76.1 \pm 25.3$ & $61.5 \pm 19.7$ & $87.7 \pm 20.9$ \\
\hline $\operatorname{AUC}_{120}(\mathrm{mg} / \mathrm{kg})$ & $203 \pm 25$ & $188 \pm 27$ & $195 \pm 20$ & $230 \pm 16$ & $212 \pm 29$ & $304 \pm 42$ & $288 \pm 45$ & $362 \pm 55$ \\
\hline
\end{tabular}

Endogenous glucose production during euglycemic clamps. $\mathrm{T}_{1 / 2}$ represents time to half-maximal suppression calculated on smoothed EGP profiles. $\mathrm{AUC}_{120}$ represents total EGP throughout the first $2 \mathrm{~h}$ of the clamp. 
Table III. Linear Regression of Insulin Effect

\begin{tabular}{lrcrr}
\hline & No glucagon & Low glucagon & Replacement glucagon & High glucagon \\
\hline$\alpha_{1}$ & $-0.0159 \pm 0.0045$ & $-0.0151 \pm 0.0030$ & $-0.0096 \pm 0.0039$ & $-0.0067 \pm 0.0027$ \\
$\alpha_{2}$ & $-0.0019 \pm 0.0035$ & $-0.0049 \pm 0.0022$ & $-0.0025 \pm 0.0020$ & $-0.0054 \pm 0.0018$ \\
Direct effect & $10.7 \pm 19.2 \%$ & $24.4 \pm 10.8 \%$ & $20.5 \pm 17.3 \%$ & $44.4 \pm 16.9 \%$ \\
\hline
\end{tabular}

Calculated $\alpha$ parameters and direct effect of insulin from multiple linear regression using the equation:

$\Delta \mathrm{EGP}=\alpha_{1} \times \Delta$ systemic insulin $+\alpha_{2} \times \Delta$ hepatic insulin.

Direct effect is equal to

$\frac{\alpha_{2}}{\alpha_{1}+\alpha_{2}} \times 100 \%$.

represent truly independent variables), the direct effects were simply 0.72 times those determined with hepatic insulin levels (i.e., $8,18,15$, and $32 \%$ for the four glucagon doses), since hepatic blood is calculated as being $72 \%$ portal and $28 \%$ arterial. Thus, the direct effect estimates obtained from multiple linear regression agree with the statistical comparisons of portal vs. systemic insulin infusion suppression of EGP: virtually no direct effect at zero glucagon, modest direct effects at low or replacement doses, and a substantial, yet secondary, direct effect of portal insulin at high glucagon.

\section{Discussion}

Despite the plethora of studies examining the metabolic effects of insulin, the mechanism whereby insulin suppresses liver glucose production remains in debate. Because insulin is secreted directly into the portal vein, and the liver extracts about half of the insulin from the portal vein, it was reasonable

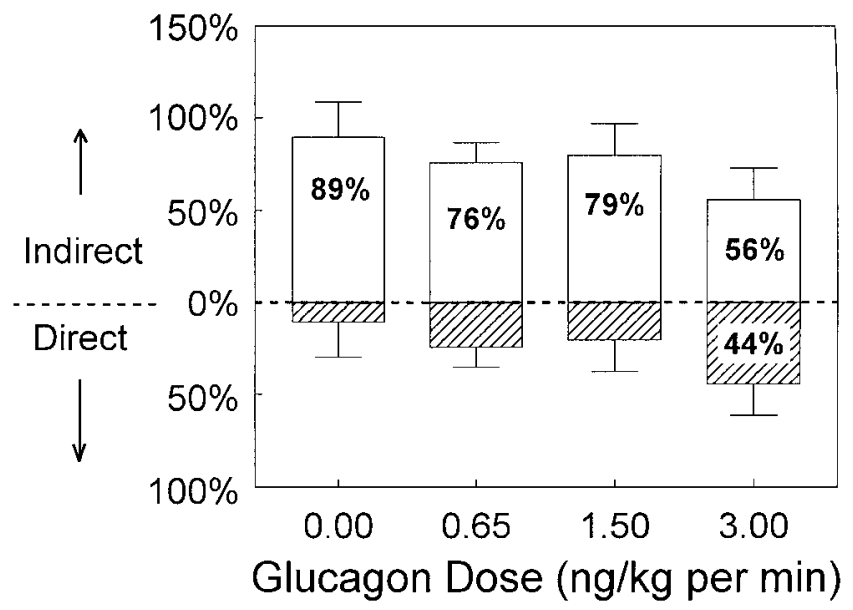

Figure 6. The direct and indirect effects of insulin to suppress EGP, shown as a percentage of total EGP suppression. The direct and indirect effects were quantified using multiple linear regression, with the equation:

$\Delta \mathrm{EGP}=\alpha_{1} \times \Delta$ systemic insulin $+\alpha_{2} \times \Delta$ hepatic insulin.

The direct effect is then equal to

$\frac{\alpha_{2}}{\alpha_{1}+\alpha_{2}} \times 100 \%$. to assume that insulin suppresses liver glucose production primarily via a direct effect on the liver. However, the inability of insulin to consistently suppress liver glucose production in vitro led Levine et al. in 1956 to hypothesize that insulin suppresses the liver through an indirect mechanism (32). Since that time, in vitro studies have succeeded in demonstrating a direct effect of insulin on the liver generally only at very high insulin levels or with elevated glucagon $(2,12,13)$. In vivo studies have had mixed results, with some $(7,10,14,15,18,20$, $21)$, but not all studies $(4,5,9)$ reporting a direct effect of insulin to suppress EGP. In contrast, two studies from our laboratory have indicated that insulin's effect to suppress EGP is primarily mediated by an indirect mechanism $(4,5)$. Since comparisons among studies may be confounded by differences in glucagon levels, and in vitro evidence suggests that the direct effect of insulin depends on glucagon $(2,12)$, the present study was undertaken to examine the direct vs. indirect effects of insulin on glucose production in vivo, in the presence of different glucagon levels. The present results show that when glucagon was suppressed but not replaced, insulin exerted a minimal direct effect, estimated at $\sim 10 \%$ of its total liver-suppressing ability (Table III, Fig. 6). Portal replacement of glucagon was associated with $\sim 25 \%$ of insulin's action being direct, yet portal insulin infusion still did not suppress EGP significantly further than systemic. The absence of significant differences between portal and systemic insulin infusions at each of the three lower glucagon doses may be due to a lack of power to detect such small effects on EGP. Only when glucagon was infused at a supranormal dose, yielding steady-state systemic levels such as those seen in starvation (33) or non-insulin-dependent diabetes mellitus (NIDDM) (34-36), did portal insulin infusion suppress EGP further than systemic infusion, and even in this case insulin's direct effect was calculated to account for only $44 \%$ of its total effect.

The ability of insulin to inhibit liver glucose production in vitro has been controversial. Yu et al. showed that insulin does not suppress gluconeogenesis in isolated fasted rat hepatocytes in the absence of glucagon, but they did report a direct insulin effect in hepatocytes from streptozotocin-induced diabetic rats under similar conditions (13). Claus et al. confirmed this finding on fasted rat hepatocytes, and further found that insulin did exhibit a direct effect when glucagon concentrations were raised (1). However, Sánchez-Gutiérrez et al. found a direct effect of insulin without glucagon in lean Zucker rat hepatocytes, but no effect on hepatocytes from obese rats (37). Several rat liver perfusion studies have either failed to detect a di- 
rect effect of insulin on glucose output (38) or confirmed the dependence on glucagon of insulin's direct effect to suppress glycogenolysis $(2,12)$. Whether these discrepancies are due to differences in animal strains, tissue isolation techniques, and/or experimental designs, it appears that in vitro, insulin's direct effect on the liver is minimal in the absence of glucagon, since concentrations as high as $10 \mathrm{nM}$ showed only modest suppression of gluconeogenesis or glycogenolysis when glucagon was not present.

Studies performed in vivo that examined the direct vs. indirect effects of insulin have also yielded conflicting results. Many investigators who have examined this system used protocols in which insulin was infused portally and peripherally at the same rate $(7,14-16,39)$. These studies are confounded by the failure to match either portal or peripheral insulin concentrations, making comparisons between these routes of administration highly problematic (compare with reference 4). Still, many of these studies found similar hypoglycemic effects of equimolar portal and peripheral insulin $(7,14,15)$. Since systemic insulin levels are inevitably lower during equimolar portal infusions due to hepatic extraction, this result implies that there is a direct effect of insulin. However, differences in glucose, glucagon, FFA, and other metabolites and counterregulatory factors confound EGP comparisons between protocols. Also due to the unmatched insulin levels, these studies were unable to report on the relative importance of the direct and indirect effects of insulin.

More recent studies have attempted to match peripheral insulin while modulating portal levels to facilitate comparisons of direct and indirect effects of insulin to suppress fasting EGP $(4,5,9,10,21)$. To do this, Ader et al. in this laboratory introduced the idea of comparing portal and peripheral insulin infusions, where the peripheral dose is one-half that of the portal, so that systemic insulin levels are matched due to the $\sim 50 \%$ hepatic extraction, while portal and hepatic insulin levels would be very different (4). Ader et al. suppressed endogenous insulin and glucagon secretion with somatostatin, and replaced glucagon with a portal infusion. Insulin was infused portally at six different doses, or systemically at half of these doses. Since EGP was suppressed similarly between portal and one-half peripheral infusions, the authors reported that the dominant effect of insulin at replacement glucagon levels was indirect. Methodological advancements have since allowed us to more accurately assess the direct effect of insulin, as in the present study, although the conclusion remains similar.

In a study by Rebrin et al., insulin was infused portally, or peripherally at half the portal rate, over several insulin doses (5). At all doses except the lowest dose couplet (1.2/0.6 pmol/ min per $\mathrm{kg} \mathrm{PO} / \mathrm{PE}$ insulin infusion), endogenous glucose production was suppressed equally between portal and peripheral infusions. However, due to hepatic extraction of $58 \%$, systemic insulin was modestly underreplaced during portal infusion. Also, at the lowest insulin dose, EGP was suppressed further with portal insulin than peripheral. The authors concluded that there are both portal and peripheral effects of insulin to suppress EGP, but the peripheral effect dominates. This conclusion is confirmed by the modest $(11 \%)$ direct effect of insulin seen at the zero glucagon dose in the present study.

A modified method was used by Sindelar and colleagues (18), who compared the effects of selective increases in portal vein and systemic insulin levels on net endogenous glucose production. The authors concluded that matched changes in portal and systemic insulin levels suppress liver glucose output to a similar degree. The effect of portal insulin may have been overestimated in the Sindelar study however, since systemic insulin levels rose $\sim 10 \%$ in the portal group, and there was a $\sim 20 \%$ drop in net hepatic glucose output in the portal control group (in which portal insulin levels did not rise). Also, the Sindelar study measured net hepatic glucose output, which includes glucose uptake by the liver, which they estimated to account for $\sim 20 \%$ of the drop in net hepatic glucose output. The authors also used tracer to measure EGP, but used methodology now known to be inaccurate (an unlabeled variable glucose infusion), which has been shown to confound this measurement due to the inconstancy of the specific activity (23, 40). When these methodological differences are taken into account, one can conclude that the results of Sindelar et al.'s study are not very different from those of the present study.

Giacca, Vranic, and colleagues performed two studies on depancreatized dogs comparing the abilities of portal, peripheral, and half-peripheral insulin infusions to suppress EGP (9, 21). In the first of these studies (9), glucagon was suppressed by insulin infusion and not replaced. The portal and one-half dose peripheral insulin infusions yielded similar systemic insulin levels and caused the same degree of EGP suppression. The study concluded that suppression of glucose production depends only on peripheral insulin levels. However, basal and steady-state glucagon levels were not matched between the portal and one-half dose peripheral protocols, and basal metabolites (alanine, FFA) were also different between these groups. In the second study (21), the same protocol was used, except that this time glucagon was infused at a high rate $(5 \mathrm{ng} /$ $\mathrm{kg}$ per min) and maintained constant. In this study, the portal infusion suppressed EGP slightly more than the one-half dose peripheral insulin infusion, implying a direct effect of insulin on the liver. When these two studies are compared, they imply that glucagon might enhance the direct effect of insulin on the liver, a finding that agrees with the present study.

In two studies by Lewis et al. $(10,20)$ done in human volunteers, tolbutamide infusions were used to stimulate portal insulin secretion, and C-peptide measurements were used to estimate the insulin secretion rate. Insulin was then infused systemically at this estimated rate. In the first study (10), glucagon was allowed to fall freely in response to the rise in insulin. Lewis concluded from this experiment that endogenous glucose production was dependent on both systemic and portal insulin levels. The glucagon suppression in Lewis' study was $\sim 30 \%$, which is between that seen in the zero and low glucagon doses of the present study. Analysis of the average results from the Lewis study by multiple linear regression using the same methods used in the present study shows the direct effect to be $17 \%$, a value between those calculated from the zero and low dose glucagon groups in the present study. A recent study by Lewis et al. (20) was performed similarly but with the addition of a low dose glucagon infusion. In this case, portal insulin secretion was more potent in suppressing EGP than equimolar systemic infusion. The authors attributed this difference to the less suppressed glucagon, and concluded that insulin's direct effect on the liver is enhanced by glucagon levels. However, both of these protocols were performed without matching either portal or systemic insulin levels, and so glucagon, FFA, and presumably any metabolites sensitive to systemic insulin levels were different between protocols. Also, portal and systemic insulin levels were different among the same experimen- 
tal groups between studies, making comparisons between the two studies difficult.

In the present study, insulin was infused portally at one rate or peripherally at half of this rate to match peripheral insulin levels while changing portal levels. Although we had previously found a hepatic insulin extraction rate of $58 \%$ (5), we did not use a $<50 \%$ portal infusion rate because of the widespread acceptance in the literature of a $50 \%$ extraction rate. However, the present group of animals exhibited a $58 \%$ extraction rate as well, causing the peripheral insulin levels during portal infusion to be $17 \%$ lower than during the systemic infusion. This undermatching would make the likelihood of missing a direct effect of insulin (type II error) greater than incorrectly identifying one (type I error), if differences in systemic insulin levels were ignored. In contrast to the slight difference in systemic levels, hepatic insulin levels were almost twice as high with portal infusion than with systemic infusion. To cope with imperfect matching, ANOVA was performed with an adjustment for systemic insulin levels. Furthermore, multiple linear regression was used to calculate the direct vs. indirect effects of insulin, which takes into account differences in systemic insulin levels. This method assumes that the suppression of EGP is a linear function of the changes in the systemic and hepatic insulin levels during the clamps. Sindelar et al. showed that incremental increases in portal and systemic insulin cause additive suppression of EGP (17), and we have shown that steady-state EGP is linearly related to systemic insulin levels in the range of this experiment (5).

To the extent that the direct effect of insulin may have been saturated during portal insulin infusion, the values obtained by linear regression would represent underestimations of the true direct effect of insulin. This could have occurred if the portal infusions were not completely mixed before entering the liver, which could cause a saturation of the direct effect of insulin in the overinsulinized lobes of the liver. However, we believe that imperfect mixing does not explain the present results. We and others have previously shown that infusions $>3 \mathrm{~cm}$ upstream from the porta hepatis of dogs are, on average, evenly distributed to the lobes of the liver $(22,41)$. Also, the $58 \%$ hepatic insulin extraction seen in these experiments argues against incomplete mixing of portally infused insulin. If the insulin infused into the portal vein did not rapidly mix with the portal blood, then it would not be expected to perfuse all parts of the liver, which might be expected to decrease, not increase hepatic insulin extraction from the expected $50 \%$.

The endogenous glucose production reported in this study includes that from both the liver and the kidney. Renal glucose production has been shown to account for up to $30 \%$ of fasting EGP and is believed to be controlled by systemic insulin levels $(11,42)$. Therefore, part of the indirect effect of insulin calculated in this study could represent suppression of the kidney, since renal glucose production should have been similarly suppressed during both portal and systemic insulin infusion. If one were to partition insulin's effects on the liver itself, one would have calculated a smaller indirect effect of insulin and therefore a proportionately larger direct effect. Thus, it is important to note that the direct and indirect effects of insulin reported in this study represent the physiologic role of insulin to suppress endogenous glucose production, and not just the effects on the liver itself.

Both the ANOVA and linear regression results show that under physiologic glucagon levels, insulin acts primarily via an indirect mechanism to suppress steady-state EGP. Even under the hyperglucagonemic conditions of the high glucagon dose, the indirect effect of insulin to suppress steady-state EGP dominates. However, neither of these methods reflects changes in EGP during the dynamic periods of the clamps. At the zero glucagon dose, the EGP profile was identical between portal and systemic insulin infusion, implying that insulin had neither a dynamic nor steady-state direct effect to suppress EGP. As the glucagon dose was increased, however, greater differences between the two curves developed, as reflected in the differences in the $\mathrm{AUC}_{120}$ values between systemic and portal insulin infusion. The well-documented, evanescent effect of glucagon to stimulate EGP was evident during this period when insulin was infused systemically at the replacement and high glucagon doses. This is believed to be primarily due to a stimulation of glycogenolysis by glucagon, which wanes over time $(29,31)$. During systemic insulin infusion at the two highest glucagon doses, EGP was actually stimulated above basal during the first hour of the clamp, whereas this effect was countered by portal insulin infusion. The large differences between the portal and systemic insulin infusions at these doses indicates that insulin has a strong direct effect to counter glucagon stimulation of EGP, although this effect is not quantified in the present study.

The glucagon doses used in the present study were designed to include the entire physiological range of glucagon levels. The high glucagon dose yielded concentrations significantly elevated from basal and comparable to those reported in NIDDM (34-36), prolonged fasting (33), and portal hypertension (43). The replacement dose, $1.5 \mathrm{ng} / \mathrm{kg}$ per min infused portally, was calculated based on pilot studies with the other three doses. The true replacement dose of glucagon has long been under debate, most likely due to the variability of glucagon radioimmunoassays caused by cross reactivity of antiglucagon antibodies to endogenous glucagon-like proteins and the rapid degradation of glucagon (44). Glucagon clearance and sensitivity have also been shown to exhibit day to day variability (30), and it is also possible that there are differences in the quality of commercially available glucagon. The glucagon used in the present study (Sigma Chemical Co.) showed good agreement with the Novo-Nordisk standards based on measurements of the infusates from each experiment (not shown). However, discrepancy between glucagon bioactivity and immunoreactivity could account for some of the variability in replacement doses calculated in different studies. The present dose of $1.5 \mathrm{ng} / \mathrm{kg}$ per min portally agrees closely with the findings of Bradley et al. (45), who determined a physiologic portal glucagon replacement dose of $2 \mathrm{ng} / \mathrm{kg}$ per min. However, the dogs in the Bradley study had a higher basal glucagon than the present study $(153 \pm 16$ vs. $90 \pm 6 \mathrm{pg} / \mathrm{ml})$, which might account for the lower replacement dose in the present study.

The mechanism of insulin's direct effect on the liver has been examined extensively in isolated hepatocytes $(1,37,46-$ 48). Insulin inhibits cAMP production caused by glucagon or epinephrine. This suppresses gluconeogenesis and glycogenolysis by lowering the activity of the cAMP-dependent protein kinase. However, insulin has also been shown to have cAMPindependent effects to inhibit cAMP-dependent protein kinase (46) and suppress gluconeogenesis (1). The present study allows for the possibility of both glucagon-dependent and -independent portal insulin effects, which could represent direct and cAMP-mediated regulation of the endogenous glucose pro- 
duction. Rebrin et al. were the first to show that the indirect effect of insulin to suppress EGP is dependent on insulin inhibition of lipolysis and the concomitant fall in FFA levels $(5,6)$. This dependence of EGP suppression on FFA suppression has since been confirmed by others (49). Free fatty acids have been shown to stimulate gluconeogenesis in both isolated hepatocytes (50), and perfused liver (51). This effect may be due to increased acetyl CoA stimulation of pyruvate carboxylase, increased NADH, or an increase in ATP production (51). Thus, evidence exists for both direct and indirect regulation of EGP at the hepatocyte.

Insulin suppression of endogenous glucose production is essential to the body's ability to restore euglycemia after a meal, and probably plays a role in maintaining euglycemia in the fasted state as well. Uncovering the mechanism of insulin action is integral to the study of insulin resistance. The precise defects occurring in insulin resistance can only be understood in the context of insulin's physiological role to lower glucose. While our results indicate that $\sim 25 \%$ of insulin's effect to suppress steady-state EGP appears to be direct in the postabsorptive, euglucagonemic state, it is clear that insulin has a direct effect to suppress glucagon stimulation of EGP, which increases with glucagon dose. Thus, the direct effect of insulin may be different in high glucagon states such as NIDDM (3436). Since glucagon is normally suppressed during a meal, it is likely that the bulk of insulin's postprandial effect is indirect. Given the minimal direct effect calculated in the present study, taken together with most in vitro and in vivo results, it seems likely that under normal conditions, insulin has at most a modest effect to directly suppress the liver in the absence of glucagon. However, in NIDDM there is often a failure of meals to suppress glucagon, and in fact, a paradoxical rise in glucagon sometimes occurs $(35,36)$, either of which might alter insulin's direct effect. It is clear that the suppression of EGP is not a simple response to insulin, but rather a complex interplay between the actions of glucagon and insulin.

\section{Acknowledgments}

The authors would like to thank the following for their helpful comments on the preparation of this manuscript: Dr. Marilyn Ader, Dr. Jang Youn, Dr. Marianthe Hamilton-Wessler, Lisa Getty, and Melvin Dea. We are also indebted to Donna M. Moore for her expert technical assistance.

This work was supported by research grants from the National Institutes of Health (DK27619 and DK29867). S.D. Mittelman is a predoctoral trainee supported by the National Institutes of Aging (T32AG-00093). Y.Y. Fu and K. Rebrin are postdoctoral fellows supported by a mentor-based grant from the American Diabetes Association.

\section{References}

1. Claus, T.H., and S.J. Pilkis. 1976. Regulation by insulin of gluconeogenesis in isolated rat hepatocytes. Biochim. Biophys. Acta. 421:246-262.

2. Mackrell, D.J., and J.E. Sokal. 1969. Antagonism between the effects of insulin and glucagon on the isolated liver. Diabetes. 18:724-732.

3. Madison, L.L., B. Combes, W. Strickland, R. Unger, and R. Adams. 1959. Symposium on the hypoglycemic agents: evidence for a direct effect of insulin on hepatic glucose output. Metab. Clin. Exp. 8:469-471.

4. Ader, M., and R.N. Bergman. 1990. Peripheral effects of insulin dominate suppression of fasting hepatic glucose production. Am. J. Physiol. 258: E1020-E1032.

5. Rebrin, K., G.M. Steil, L. Getty, and R.N. Bergman. 1995. Free fatty acid as a link in the regulation of hepatic glucose output by peripheral insulin. Diabetes. 44:1038-1045.
6. Rebrin, K., G.M. Steil, S.D. Mittelman, and R.N. Bergman. 1996. Causal linkage between insulin suppression of lipolysis and suppression of liver glucose output in dogs. J. Clin. Invest. 98:741-749.

7. Ishida, T., Z. Chap, J. Chou, R.M. Lewis, C.J. Hartley, M.L. Entman, and J.B. Field. 1984. Effects of portal and peripheral venous insulin infusion on glucose production and utilization in depancreatized, conscious dogs. Diabetes. 33: 984-990.

8. Prager, R., P. Wallace, and J.M. Olefsky. 1987. Direct and indirect effects of insulin to inhibit hepatic glucose output in obese subjects. Diabetes. 36:607-611.

9. Giacca, A., S.J. Fisher, Z.Q. Shi, R. Gupta, H. Lavina, A. Lickley, and M. Vranic. 1992. Importance of peripheral insulin levels for insulin-induced suppression of glucose production in depancreatized dogs. J. Clin. Invest. 90:17691777

10. Lewis, G.F., B. Zinman, Y. Groenewoud, M. Vranic, and A. Giacca. 1996. Hepatic glucose production is regulated both by direct hepatic and extrahepatic effects of insulin in humans. Diabetes. 45:454-462.

11. Cersosimo, E., R.L. Judd, and J.M. Miles. 1994. Insulin regulation of renal glucose metabolism in conscious dogs. J. Clin. Invest. 93:2584-2589.

12. Glinsmann, W.H., and G.E. Mortimore. 1968. Influence of glucagon and 3', 5'-AMP on insulin responsiveness of the perfused rat liver. Am. J. Physiol. 215:553-559.

13. Yu, B., S. Pugazhenthi, and R.L. Khandelwal. 1994. Effects of metformin on glucose and glucagon regulated gluconeogenesis in cultured normal and diabetic hepatocytes. Biochem. Pharmacol. 48:949-954.

14. Cherrington, A.D., W.W. Lacy, P.E. Williams, and K.E. Steiner. 1983. Failure of somatostatin to modify effect of glucagon on carbohydrate metabolism in the dog. Am. J. Physiol. 244:E596-E602.

15. Stevenson, R.W., J.A. Parsons, and K.G.M.M. Alberti. 1983. Effect of intraportal and peripheral insulin on glucose turnover and recycling in diabetic dogs. Am. J. Physiol. 244:E190-E195.

16. Griffen, S.C., S.M. Russell, L.S. Katz, and C.S. Nicoll. 1987. Insulin exerts metabolic and growth-promoting effects by a direct action on the liver in vivo: clarification of the functional significance of the portal vascular link between the beta cells of the pancreatic islets and the liver. Proc. Natl. Acad. Sci. USA. 84:7300-7304.

17. Sindelar, D.K., C.A. Chu, A. Venkatakrishnan, P. Venson, and A.D Cherrington. 1996. The interaction between increments in peripheral and portal vein insulin in regulating hepatic glucose production. Diabetes. 45:167a. (Abstr.)

18. Sindelar, D.K., J.H. Balcom, C.A. Chu, D.W. Neal, and A.D. Cherrington. 1996. A comparison of the effects of selective increases in peripheral or portal insulin on hepatic glucose production in the conscious dog. Diabetes. 45: 1594-1604.

19. Polonsky, K., J. Jaspan, D. Emmanouel, K. Holmes, and A.R. Moossa. 1983. Differences in the hepatic and renal extraction of insulin and glucagon in the dog: evidence for saturability of insulin metabolism. Acta Endocrinol. 102: $420-427$.

20. Lewis, G.F., M. Vranic, and A. Giacca. 1997. Glucagon enhances the direct suppressive effect of insulin on hepatic glucose production in humans. Am. J. Physiol. 272:E371-E378.

21. Giacca, A., S.J. Fisher, R.H. McCall, Z.Q. Shi, and M. Vranic. 1997. Direct and indirect effects of insulin in suppressing glucose production in depancreatized dogs: role of glucagon. Endocrinology. 138:999-1007.

22. Bergman, R.N., M. Ader, D.T. Finegood, and G. Pacini. 1984. Extrapancreatic effect of somatostatin infusion to increase glucose clearance. Am. J. Physiol. 247:E370-E379.

23. Finegood, D.T., R.N. Bergman, and M. Vranic. 1987. Estimation of endogenous glucose production during hyperinsulinemic-euglycemic glucose clamps: comparison of unlabeled and labeled exogenous glucose infusates. Diabetes. 36:914-924

24. Zambon, A., S.I. Hashimoto, and J.D. Brunzell. 1993. Analysis of techniques to obtain plasma for measurement of levels of free fatty acids. J. Lipid Res. 34:1021-1028.

25. Katz, J., F. Okajima, M. Chenoweth, and A. Dunn. 1981. The determination of lactate turnover in vivo with $3 \mathrm{H}$ and 14C-labeled lactate: the significance of sites of tracer administration and sampling. Biochem. J. 194:513-524.

26. Greenway, C.V., and R.D. Stark. 1971. Hepatic vascular bed. Physiol. Rev. 51:23-65.

27. Hother-Nielsen, O., J.E. Henriksen, P. Staehr, and H. Beck-Nielsen. 1995. Labeled glucose infusate technique in clamp studies. Is precise matching of glucose specific activity important? Endocrinol. Metab. 2:275-287.

28. Fisher, S.J., Z.Q. Shi, L.A. Lickley, S. Efendic, M. Vranic, and A. Giacca. 1996. A moderate decline in specific activity does not lead to an underestimation of hepatic glucose production during a glucose clamp. Metab. Clin. Exp. 45:587-593.

29. Cherrington, A.D., P.E. Williams, G.I. Shulman, and W.W. Lacy. 1981. Differential time course of glucagon's effect on glycogenolysis and gluconeogenesis in the conscious dog. Diabetes. 30:180-187.

30. Bradley, D.C., and R.N. Bergman. 1992. Hepatic glucagon sensitivity and fasting glucose concentration in normal dogs. Am. J. Physiol. 262:E539E545.

31. Magnusson, I., D.L. Rothman, D.P. Gerard, L.D. Katz, and G.I. Shul- 
man. 1995. Contribution of hepatic glycogenolysis to glucose production in humans in response to a physiological increase in plasma glucagon concentration. Diabetes. 44:185-189.

32. Levine, R., and I.B. Fritz. 1956. The relation of insulin to liver metabolism. Diabetes. 5:209-219.

33. Marliss, E.B., T.T. Aoki, R.H. Unger, J.S. Soeldner, and G.F.J. Cahill. 1970. Glucagon levels and metabolic effects in fasting man. J. Clin. Invest. 49: 2256-2270.

34. Baron, A.D., L. Schaeffer, P. Shragg, and O.G. Kolterman. 1987. Role of hyperglucagonemia in maintenance of increased rates of hepatic glucose output in type II diabetics. Diabetes. 36:274-283.

35. Lefebvre, P.J., and A.S. Luyckx. 1979. Glucagon and diabetes: a reappraisal. Diabetologia. 16:347-354.

36. Gerich, J.E. 1977. On the causes and consequences of abnormal glucagon secretion in human diabetes mellitus. In Glucagon: Its Role in Physiology and Clinical Medicine. P.P. Foa, J.S. Bajaj, and N.L. Foa, editors. Springer-Verlag, New York. 617-641.

37. Sánchez-Gutiérrez, J.C., J.A. Sánchez-Arias, C.G. Lechuga, J.C. Valle, B. Samper, and J.E. Feliu. 1994. Decreased responsiveness of basal gluconeogenesis to insulin action in hepatocytes isolated from genetically obese (fa/fa) Zucker rats. Endocrinology. 134:1868-1873.

38. Williamson, J.R., A. Garcia, A.E. Renold, and G.F.J. Cahill. 1966. Studies on the perfused rat liver I: effects of glucagon and insulin on glucose metabolism. Diabetes. 15:183-187.

39. Rizza, R.A., R.E. Westland, L.D. Hall, G.S. Patton, M.W. Haymond, A.H. Clemens, J.E. Gerich, and F.J. Service. 1981. Effect of peripheral versus portal venous administration of insulin on postprandial hyperglycemia and glucose turnover in alloxan-diabetic dogs. Mayo Clin. Proc. 56:434-438.

40. Hother-Nielsen, O., A. Mengel, J. Moller, O. Rasmussen, O. Schmitz, and H. Beck-Nielsen. 1992. Assessment of glucose turnover rates in euglycemic clamp studies using primed-constant $[3-3 \mathrm{H}]$ glucose infusion and labeled or unlabeled glucose infusates. Diabetic Med. 9:840-849.
41. Greenway, C.V., and G. Oshiro. 1972. Intrahepatic distribution of portal and hepatic arterial blood flows in anaesthetized cats and dogs and the effects of portal occlusion, raised venous pressure and histamine. J. Physiol. 227:473-485.

42. Stumvoll, M., U. Chintalapudi, G. Perriello, S. Welle, O. Gutierrez, and J.E. Gerich. 1995. Uptake and release of glucose by the human kidney: postabsorptive rates and responses to epinephrine. J. Clin. Invest. 96:2528-2533.

43. Pak, J.M., and S.S. Lee. 1994. Glucagon in portal hypertension. J. Hepatol. 20:825-832.

44. Borghi, V.C., B.L. Wajchenberg, and R.H. Albuquerque. 1984. Evaluation of a sensitive and specific radioimmunoassay for pancreatic glucagon in human plasma and its clinical application. Clin. Chim. Acta. 136:39-48.

45. Bradley, D.C., and R.N. Bergman. 1992. Restoration of stable metabolic conditions during islet suppression in dogs. Am. J. Physiol. 262:E532-E538.

46. Gabbay, R.A., and H.A. Lardy. 1984. Site of insulin inhibition of cAMPstimulated glycogenolysis: cAMP-dependent protein kinase is affected independent of cAMP changes. J. Biol. Chem. 259:6052-6055.

47. Boyd, M.E., E.B. Albright, D.W. Foster, and J.D. McGarry. 1981. In vitro reversal of the fasting state of liver metabolism in the rat. J. Clin. Invest. 68:142-152.

48. Iynedjian, P.B., S. Marie, A. Gjinovci, B. Genin, S.P. Deng, L. Buhler, P. Morel, and G. Mentha. 1995. Glucokinase and cytosolic phosphoenolpyruvate carboxykinase (GTP) in the human liver. Regulation of gene expression in cultured hepatocytes. J. Clin. Invest. 95:1966-1973.

49. Sindelar, D.K., C.A. Chu, M. Rohlie, D.W. Neal, L.L. Swift, and A.D. Cherrington. 1997. The role of fatty acids in mediating the effects of peripheral insulin on hepatic glucose production in the conscious dog. Diabetes. 46:187-196.

50. Morand, C., C. Remesy, and C. Demigne. 1993. Fatty acids are potent modulators of lactate utilization in isolated hepatocytes from fed rats. Am. J. Physiol. 264:E816-E823.

51. Williamson, J.R., R.A. Kreisberg, and P.W. Felts. 1966. Mechanism for the stimulation of gluconeogenesis by fatty acids in perfused rat liver. Proc Natl. Acad. Sci. USA. 56:247-254. 